\title{
¿Todas las pestes la peste? Individuo, destino y modernidad en el Diario del año de la peste (1722)
}

\section{Carolina Martínez'}

Resumen: El artículo examina los efectos de la gran plaga de Londres (1665) tal como fue narrada por Daniel Defoe en Diario del año de la peste (1772) a partir de dos ejes de análisis: las tensiones entre individuo y comunidad en la temprana modernidad y su carácter propedéutico a la luz de los acontecimientos actuales.

Palabras clave: Daniel Defoe. plaga. Londres. modernidad temprana.

\section{All Plagues the Plague? Individual, Fate and Modernity in A Journal of the Plague Year (1722)}

\begin{abstract}
The article examines the effects of the great plague of London (1665) as narrated by Daniel Defoe in his Journal of the Plague Year (1772) from two axes of analysis: the tensions between individual and community in the early-modern period and its propaedeutic character in the light of current events.
\end{abstract}

Keywords: Daniel Defoe. Plague. London. Early Modern Period.

I Es doctora en Historia por la Universidad de Buenos Aires y la Universidad de Paris 7 Diderot. Como investigadora del CONICET en Argentina, actualmente se dedica al estudio de la expansión ultramarina europea (siglos XVI-XVIII) desde las perspectivas abiertas por la historia cultural. En el primer semestre de 2020 fue profesora invitada del Programa de Pós-Graudação em História da Arte de la UERJ. Vínculo institucional: Consejo Nacional de Investigaciones Científicas y Técnicas (CONICET) - Universidad Nacional de San Martín (UNSAM), Laboratorio de Investigación en Ciencias Humanas (LICH), Escuela de Humanidades, Campus Miguelete Martín de Irigoyen 3100, 1600 San Martín, Provincia de Buenos Aires - Argentina. E-mail: carolina.martinez@unsam.edu.ar. ORCID: https://orcid.org/00000002-5692-0995. Buenos Aires, Argentina. 
New readers make new texts, and their meanings are a function of their new forms.

D. F. McKenzie ${ }^{2}$

En Todos los fuegos el fuego, uno de los ocho cuentos del libro homónimo de Julio Cortázar, el relato presenta la historia de dos parejas de amantes cuyo trágico final está signado por las llamas. ${ }^{3}$ Aunque distanciados en el tiempo, pues los avatares de la primera pareja transcurren en la antigua Roma y los de la segunda en una París contemporánea, el devenir de los amantes es narrado en paralelo, de forma tal que la suerte de ambas parejas pareciera imbricarse en una única historia, acaso universal, cuyo destino es también el mismo. Tanto el título de la obra cuanto el ejercicio literario y la consecuente reflexión a la que el cuento invita parecieran reverberar en nuestra vivencia e interpretación de los acontecimientos actuales. ¿Puede la experiencia de una peste pasada funcionar como guía para la vivencia de la situación sanitaria actual? ¿Son todas las pestes la peste? La pandemia así como los efectos que esta provoca en la percepción de la vida y la muerte, han llevado a procurar en experiencias pasadas, pero sobre todo en la literatura, puntos de comparación o referencias sobre cómo las plagas fueron atravesadas en tiempos otros que los actuales. En este sentido, las percepciones de la peste presentes en textos como el Decamerón, escrito por Giovanni Bocaccio hacia 1353, La máscara de la muerte roja (The Masque of the Red Death), publicada por Edgar Alan Poe en 1842, o La Peste, elaborada por Albert Camus en 1947, han cobrado actualidad no por los textos en sí mismos sino porque su contenido ha sido resignificado en función de la pandemia actual. ${ }^{4}$

En relación con lo antedicho, conviene recordar las reflexiones de Roger Chartier en torno a los sentidos variables de una obra a través del tiempo. En un texto ya clásico en el área de la historia cultural, el historiador

2 McKENZIE, Donald F. Bibliography and the Sociology of Texts: Panizzi Lectures 1985. Londres: The British Library, 1986, p. 20, citado en CHARTIER, Roger. Le monde comme représentation. Annales. Économies, Sociétés, Civilisations, París, Año 44, n. 6, 1989, p. 1510. [Nuevos lectores hacen nuevos textos y sus significados son una función de sus nuevas formas.] Traducción de la autora.

3 CORTÁZAR, Julio. Todos los fuegos el fuego. Buenos Aires: Editorial Sudamericana, 1966.

4 En el caso de Boccaccio, los orígenes de la historia están directamente vinculados al brote de peste negra que azotó a las ciudades del norte de Italia en 1348. Poe y Camus, en cambio, parecieran haberse inspirado en los efectos causados por las epidemias de cólera en los siglos XIX y XX. 
francés afirmaba que "producidas en una esfera específica, el campo artístico e intelectual, que tiene sus reglas, sus convenciones, sus jerarquías, las obras se escapan y toman densidad peregrinando, a veces en períodos de larga duración, a través del mundo social". ${ }^{5}$ Chartier se interesaba entonces en la complejidad de los procesos de recepción, que otorgaban a las obras "significaciones plurales y móviles" a partir de los contextos en los que estas eran apropiadas e interpretadas. Deudor de los trabajos del bibliógrafo neozelandés Donald F. McKenzie sobre la historia del libro y la lectura, Chartier refrendaba los postulados del primero, para quien "nuevos lectores hacen nuevos textos". ${ }^{6}$ Es a partir de esta última premisa que el presente artículo busca reflexionar sobre el Diario del año de la peste (A Journal of the Plague Year), escrito por Daniel Defoe hacia 1722 (Figura 1) a partir de la gran plaga que azotó a la ciudad de Londres en 1665. El objetivo es, en consecuencia, doble pues si por un lado examina las tensiones entre individuo y comunidad en la temprana modernidad, también se detiene en su carácter propedéutico a la luz de los acontecimientos actuales. En las siguientes páginas se analizarán, entonces, tanto el contexto de su escritura cuanto la incidencia de este texto en particular dentro del corpus literario relativo a pestes y plagas.

Escrito por Daniel Defoe en 1722 pero situado en el contexto de la gran peste de Londres ocurrida entre 1665 y 1666, el Diario del año de la peste se presenta como un ejercicio de indagación personal acerca de los efectos de la peste negra en la capital inglesa $y$, en consecuencia, en el destino individual y colectivo de sus habitantes. Al comenzar su diario el protagonista explica:

He anotado este asunto tan detalladamente porque tal vez mi historia pueda resultar útil a quienes vengan detrás de mí, si alguna vez se vieran sometidos a la misma angustia y a la misma opción; por esta razón deseo que esta narración sea, más que una historia de mis actos, una guía para los de aquellos a quienes muy poco puede importar lo que fue de mí.

5 CHARTIER, Roger. El mundo como representación. Estudios sobre historia cultural. Barcelona: Gedisa, 2005, p. XI.

6 La cita corresponde al epígrafe y fue incluida por Chartier en Le monde comme représentation (op. cit.), artículo que diera origen al libro homónimo.

7 DEFOE, Daniel, Diario del año de la peste, 1722, p. 6. Disponible en: https://www.biblioteca.org.ar/libros/153823.pdf Acceso: 10 de agosto de 2020. 
Figura 1

Portada de la primera edición inglesa del Diario del año de la peste, publicada en Londres en $1722^{8}$
A.

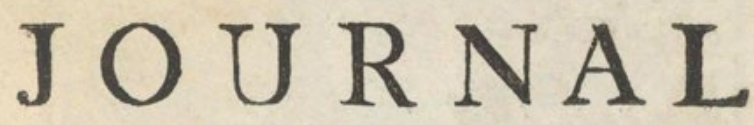

OF TH E 诌lagute 区ear:

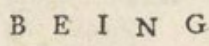

Obfervations or Memorials, Of the moft Remarkable OCCURRENCES,

As well

PUBLICK as PRIVATE, Which happened in

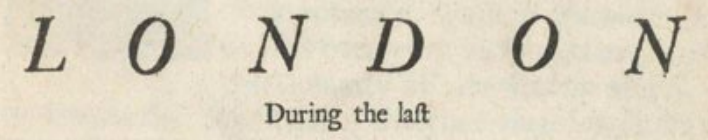

GREAT VISITATION In 1665 .

Written by a Citizen who continued all the while in London. Never made publick before

$L O N \quad \mathcal{D} O \quad N$ :

Printed for $E$. Nutt at the Royal-Exchange; 7. Roberts in Warwick-Lane; A. Dodd without Temple-Bar; and 7. Graves in St. Fames's-freet. 1722.

A su vez, el hecho de que el propio texto se presente como una guía para futuras pestes, resulta una propuesta de especial relevancia para reflexionar en torno a su posible "utilidad" a la luz de la pandemia

8 La obra se presentó al público como escrita por el anónimo H. F. y, según ha señalado F. Bastian, fue recién en 1780 que se atribuyó su autoría Daniel Defoe. Véase BASTIAN, Frank. Defoe's Journal of the Plague Year Reconsidered. The Review of English Studies, New Series, vol. 16, n. 62, mayo, 1965, p. 151. 
actual. La intención de elaborar una obra que permitiera reflexionar sobre los efectos de la plaga en Londres pero a la vez resultara una lectura edificante frente a pestes futuras ya había sido expresado por Defoe en Preparativos oportunos para una peste (Due Preparations for the Plague, as well for Soul as Body), obra sobre el mismo tema que el escritor publicara un mes antes del Diario en $1722 .{ }^{9}$ La supuesta supervivencia de dichas anotaciones (pues en el caso del diario se trata de un relato apócrifo) se vuelve aún más verosímil si se tiene en cuenta que, tal como ha señalado Andrés Gattinoni en un ensayo reciente, este tipo de escrito "remitía a una práctica fundamental de la tradición religiosa en la que se había criado Defoe". En efecto, constituía un ejercicio diario de aquellos que adscribían a la fe reformada anotar sus acciones y emociones con el objetivo de llevar cuenta de los progresos de su alma. ${ }^{10}$

Ahora bien, más allá de que fuese escrito décadas después de los acontecimientos, las reflexiones narradas en el diario solo pueden comprenderse plenamente si se toman en cuenta algunos datos sobre la peste de Londres de 1665. En principio, fue la peor plaga en Inglaterra después de la peste negra o peste bubónica ocurrida en 1348. La ciudad de Londres perdió un cuarto de su población, que en aquella época era el equivalente a cerca de 100.000 vidas. Al igual que sus antecesoras, la peste se transmitía a través de las pulgas de las ratas negras y un treinta por ciento de las personas infectadas podían morir en una o dos semanas. Los londinenses del período, sin embargo, no tenían noción de las causas de transmisión de la enfermedad. Pues, según ha señalado Jean Delumeau en su histórico trabajo sobre las múltiples manifestaciones del miedo en Occidente, antes que temer o percibir cuál era el papel de la pulga en el contagio, primó entre los contemporáneos el temor a la transmisión interhumana. ${ }^{11}$

9 DEFOE, Daniel, Due Preparations for the Plague. In: DEFOE, Daniel. The works of Daniel Defoe. New York: G. D. Sproul, 1908.

10 GATTINONI, Andrés. Diario del año de la pandemia. Revista Nueva Sociedad, Buenos Aires, (edición digital) marzo de 2020, p. 4. Disponible en: https://nuso.org/articulo/ literatura-pandemia-peste-coronavirus-defoe-bocaccio-shakespeare/ Acceso: 20 de abril de 2020.

11 DELUMEAU, Jean. El miedo en Occidente (Siglos XIV-XVIII) Una ciudad sitiada. Madrid: Taurus, 1989 [1978], p. 161. 
Al constatar la letalidad del brote, aquellos que disponían de los medios necesarios huyeron de la ciudad. De tal forma, en julio de 1665 el rey Carlos II y su corte se instalaron primero en Hampton Court y luego en Oxford. Por su parte, el Parlamento también comenzó a sesionar en esta última ciudad a partir de octubre. Las fronteras internacionales se cerraron, pues se consideraba que la peste había provenido de Holanda, y el comercio entre Londres y otras ciudades de Inglaterra también fue prohibido. Además de estas medidas, fue decretado que cualquier casa en donde la peste hubiese sido identificada debía cerrarse por cuarenta días con la familia allí dentro, marcada con una cruz y custodiada por vigías.

Tal como fue señalado en los párrafos precedentes, el Diario del año de la peste es, en principio, un relato ficticio en el que se reconstruyen los acontecimientos de $1665 .{ }^{12}$ Es posible, sin embargo, que Defoe se basara en la vida de su tío Henry Foe (de allí las iniciales H. F. con las que "firma" el texto), que sí había vivido la peste en 1665 y, al igual que el "autor" del diario, era un talabartero de Whitechapel. Según lo señala al comienzo del texto, aun pudiendo huir, el protagonista anónimo decide quedarse en la ciudad de Londres desde que se desencadena la epidemia. ${ }^{13}$ En calidad de testigo privilegiado recorre la ciudad, desde los barrios más pobres hasta la City, los cementerios, pubs, iglesias y la orilla del río Támesis, narrando las atrocidades producidas por la plaga (Figura 2).

Pero, ¿qué factores explican el éxito de la obra? En primer lugar, la plaga de 1665 había provocado un crecimiento en la publicación de distintos textos sobre ella, que muy probablemente ayudaron a Defoe a reconstruir un relato verosímil más de medio siglo después de los acontecimientos. ${ }^{14}$ Además

12 Un documento histórico del período es, en cambio, el diario del funcionario y político inglés Samuel Pepys. Las entradas hechas entre junio y diciembre de 1665 resultan vívidas anotaciones sobre los efectos de la peste entre el estrato más alto de la población en Londres. Véase PEPYS, Samuel. The Diary of Samuel Pepys M.A. F.R.S. Clerk $f$ the Acts and Secretary to the Admiralty transcribed from the shorthand manuscript in the Pepysian Library Magdalene College Cambridge by the Rev. Mynors Bright M.A. Late Fellow and President of the College (Unabridged) with Lord Braybrook's Notes. Londres: George Bell \& Sons York St. Covent Garden, 1893.

13 Por el contrario, así como las cerca de 200.000 personas que abandonaron la capital londinense hacia 1665 , el hermano del protagonista junto con su familia parte rumbo a la campiña inglesa.

14 Tal como ha señalado Kathleen Miller, la plaga había llevado a la publicación de múltiples textos sobre ella: panfletos médicos, proclamaciones políticas, tratados científicos o religiosos, escritos de vida y correspondencia. MILLER, Kathleen. The Literary Culture of 


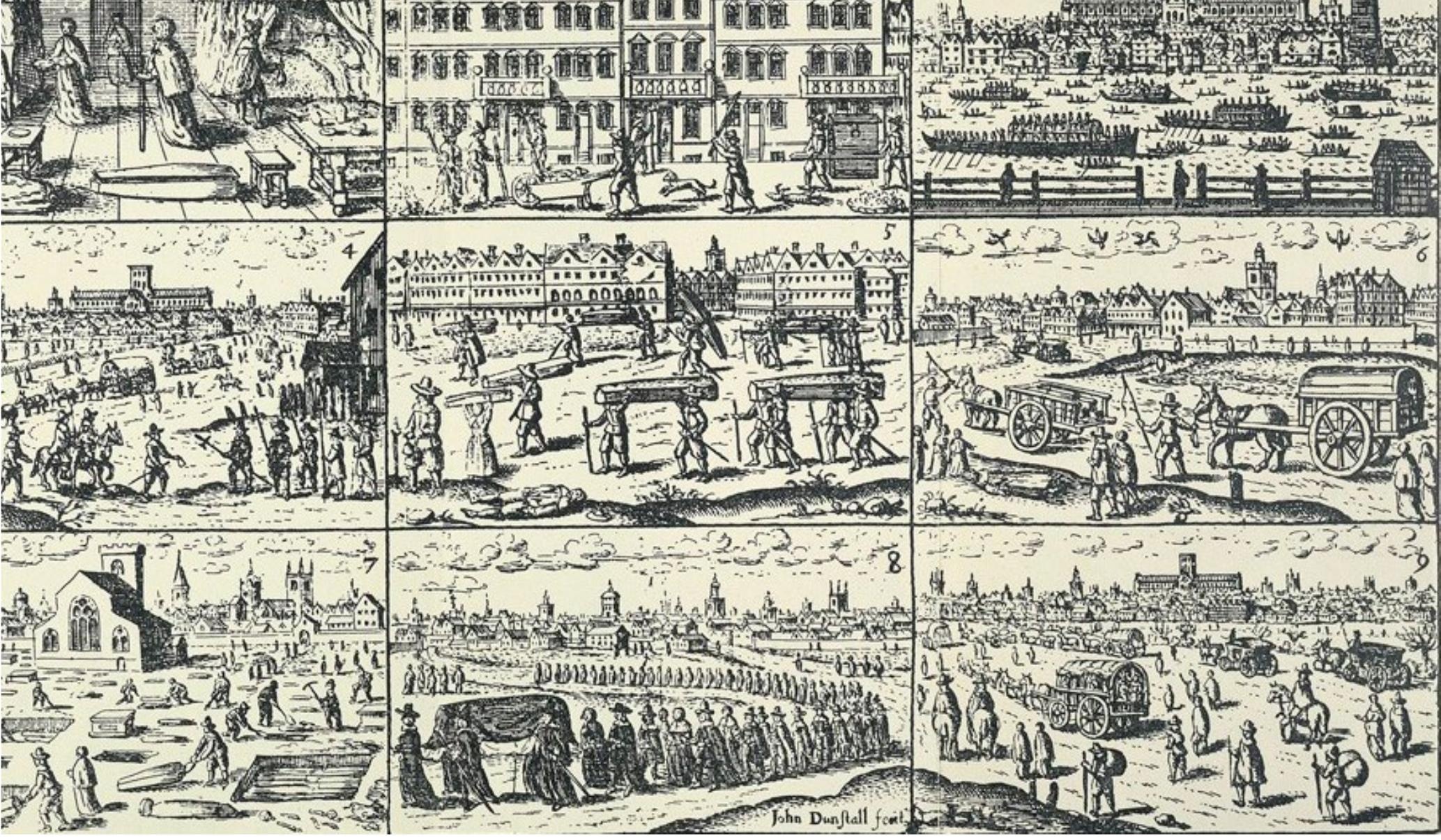

Figura 2

Grabado anónimo

Pictorial depiction of the Great Plague, 1665

En él se observan escenas corrientes en la ciudad azotada por la peste

Fuente: Museum of London/Heritage Images/Getty Images de esto, Defoe, quien ya era reconocido por su trabajo como panfletista y periodista, conoce las exigencias de un nuevo público lector, fascinado por las crónicas y los relatos periodísticos recientemente aparecidos en la prensa. También es posible que el éxito de Diario de un año de la peste se debiera a la narración en primera persona, que refleja tanto la introspección individualista del protagonista cuanto su perspectiva autónoma. ${ }^{15}$ Tal como fue señalado en los párrafos precedentes, la formación puritana de Defoe hace que el diario cumpla la función de ser una autobiografía espiritual, además de narrar muchas otras historias en su contacto con la epidemia en Londres. Sobre su intención de dar cuenta de todo aquello que sucedió en 1665, el protagonista anónimo indica:

Los intervalos los empleaba en leer y en poner por escrito las cosas de mi vida diaria; de aquellas notas extraje después la mayor parte de este trabajo, que sólo es un conjunto de observaciones efectuadas fuera de mi casa. Lo que escribí acerca de mis meditaciones lo he reservado para mi uso personal; deseo que nunca se haga público, con ningún pretexto. ${ }^{16}$

Plague in Early Modern England. London: Palgrave Macmillan, 2016, pp. 1-2.

15 KELLY, Caitlin L. Private Meditations and Public History in Daniel Defoe's A Journal of the Plague Year. The Explicator, vol. 71, n. 1, 2013, pp. 52.

16 DEFOE, Daniel. Diario del año..., op. cit., p. 39. 
Defoe construye así un relato cuasi-histórico, basado en informaciones específicas, un estilo periodístico y la narración en primera persona. Todos estos factores potenciaron su éxito editorial, además del hecho de que poco antes de que se publicara la obra, en la ciudad de Marsella se había detectado un nuevo y violento episodio de peste, lo que aumentó el interés por el texto de Defoe. En efecto, si a comienzos del siglo XVIII Marsella tenía aproximadamente 100.000 habitantes; la epidemia de 1720 se cobró aproximadamente 50.000 vidas de aquella ciudad y sus alrededores. ${ }^{17} \mathrm{La}$ razón por la que Defoe publicara el Diario del año de la peste poco después del brote originado en Marsella bien pudo haber sido comercial. ${ }^{18} \mathrm{De}$ hecho, ese mismo año también publicó los ya mencionados Preparativos oportunos para una peste, en donde daba cuenta de los recaudos espirituales y materiales que debían tomarse para enfrentarse a la plaga.

A la vez, por tratarse de una crónica sobre la desesperación y la soledad, el Diario del año de la peste bien podría ser considerado una continuación de Robinson Crusoe, novela que Defoe escribiera tres años antes, en $1719 .{ }^{19}$ Así como el náufrago Crusoe, el anónimo protagonista del Diario es un individuo en crisis ${ }^{20}$ y ambos experimentan la "soledad radical" producto de las catástrofes. ${ }^{21} \mathrm{Al}$ recorrer las calles de Londres y observar la devastación causada por la plaga en términos del sufrimiento de su población y la pérdida de vidas humanas, el anónimo H. F. reflexiona sobre las acciones individuales de los hombres y se pregunta sobre el papel de la Providencia divina en el destino humano. Por ello, en la opinión de Rogelio C. Paredes, tanto Robinson como el apócrifo autor del diario "se interrogan sobre lo mismo: la desdicha humana y sus causas, verosímiles o no, en el error y la culpa individual".22

En cuanto al carácter propedéutico del Diario, el examen atento de algunas diferencias y semejanzas respecto de la pandemia en tiempos actuales será el

17 DELUMEAU, op. cit., p. 160.

18 DEFOE, Daniel. The Works of Daniel Defoe..., op. cit.

19 PAREDES, Rogelio. Pasaporte a la utopía: literatura, individuo y modernidad en Europa (1680-1780). Buenos Aires: Miño y Dávila editores, 2003, p. 74.

20 KELLY, op. cit., p. 53: "the Journal initially appears to fit the same mold as Defoe's other novels by presenting an individual in crisis”. [...al comienzo. el diario pareciera encajar en el mismo molde que las otras novelas de Defoe, al presentar a un individuo en crisis.]

21 PAREDES, op. cit., p. 73.

22 Ibid., p. 84: "Tanto Robinson como el autor anónimo del Diario se interrogan sobre lo mismo: la desdicha humana y sus causas, verosímiles o no, en el error y la culpa individual." 
punto de partida para reflexionar en torno a la incidencia de la peste en las sociedades temprano modernas. En principio, son dos las características de esta última las que, a primera vista, más la distancian de contextos sociales y culturales actuales. Se trata, en primer lugar, de la disolución de los espacios públicos y de las diferencias sociales allí presentes. ${ }^{23}$ En segundo lugar, debe mencionarse la importancia de la Providencia como factor explicativo de la salvación individual.

En lo que refiere al primer punto mencionado, una de las características más sobresalientes del Diario es la confirmación, a través del protagonista, del alcance universal de la muerte en tiempos de peste. Defoe describe tanto la disolución de los espacios de sociabilidad pública existentes, cerrados para impedir la propagación de la enfermedad, cuanto la abertura de fosas comunes donde ricos y pobres terminan sus días igualmente. En este sentido, destaca la disolución de las distinciones sociales, materiales y culturales de una Inglaterra atormentada por la plaga. En su diario, la imposibilidad de practicar los ritos funerales tradicionales y en consecuencia distinguir socialmente a quienes podían afrontar los costos de las exequias de quienes no es descripta por el anónimo autor de la siguiente forma:

El carro transportaba dieciséis o diecisiete cuerpos; algunos envueltos en sábanas de lino, algunos en harapos, y unos pocos que estaban desnudos o se habían sacudido tanto que lo que los cubría cayó del carro durante la descarga y llegaron tan desnudos como el resto. Pero la indecencia del asunto no les importaba demasiado a ellos ni a cualquier otro, puesto que estaban muertos e iban a confundirse en la tumba común de la humanidad, como podemos llamarla, porque aquí no se hacían diferencias: pobres y ricos iban juntos. ${ }^{24}$

Defoe confirma la supresión de las divisiones sociales en los espacios públicos cuando afirma que la necesidad de los habitantes de encontrar consuelo en las iglesias sobrepasaba cualquier incomodidad causada por el hecho de compartir el mismo espacio de culto con fieles de procedencia variada. En el peor momento de la peste, señala el protagonista:

La gente buscaba estar en compañía, y era sorprendente verla ir en multitud a las iglesias. Ya nadie se preocupaba por quién se sentaba al lado, ni por alguna emanación desagradable ni por el estado de su vecino. Todos se consideraban cadáveres y

23 Ibid., p. 87: "La disolución de los espacios públicos, de los lugares donde los hombres se hablan, se reconocen y se asignan sus posiciones en el cuadro social, y consecuentemente, del reconocimiento colectivo de la condición material, espiritual, social y laboral de los individuos se enfatiza en todas las crónicas de las pestes a lo largo de la modernidad."

24 DEFOE, Diario del año..., op. cit., p. 32. 
acudían a los templos sin la menor inquietud y se sentaban juntos, como si su vida no tuviera valor alguno en comparación con el deber con que debían cumplir allí. ${ }^{25}$

La disolución de las jerarquías sociales es, sin embargo, aparente, pues no resiste el advenimiento de una nueva era de paz. De hecho, es en el mismo texto que Defoe apunta que, una vez terminada la plaga, las diferencias de clase y la demarcación de los espacios sociales fueron rápidamente restauradas. ${ }^{26}$

En cuanto al papel de la Providencia en el destino del hombre, el Diario de Defoe (así como otras obras del autor) parece expresar la tensión entre aquella y el destino individual de los hombres. ${ }^{27}$ Tal como son narradas en la obra, las tensiones entre misticismo y realismo, o entre la verdad revelada y las convicciones resultantes de la iniciativa personal, evidencian la coexistencia (muchas veces conflictiva) entre tradición y modernidad. En efecto, más allá de que Defoe rechace las profecías astronómicas y denuncie el uso de pociones mágicas para curar la plaga, no duda en afirmar que fue la "mano de Dios" la que causó y resolvió el brote de peste que azotó a la ciudad. Según el protagonista, el desarrollo de la plaga en Londres no es más que una gran punición divina, cuyo destino es castigar las culpas individuales. ${ }^{28}$ En consecuencia, así como para $\mathrm{H}$. F. la plaga comienza por obra y gracia del Creador, también termina debido a su intervención. En efecto, hacia el final del libro el anónimo autor explica que fue por causa de su "mano Divina" que el sufrimiento de Londres acabó:

En medio de aquella terrible aflicción, en momentos en que la situación de Londres era verdaderamente desastrosa, Dios quiso desarmar a su enemigo mediante la inmediata intervención de su Mano. El veneno fue extraído de su mordedura. ¡Oh, maravilla! ¡Hasta los médicos se sintieron sorprendidos! ${ }^{29}$

25 Ibid., p. 81.

26 En términos del anónimo H. F.: “...excepción hecha de unas pocas familias y de ciertos casos particulares, cabe reconocer que las costumbres generales fueron lo que habían sido antes: muy poca diferencia pudo comprobarse." Ibid., p. 91.

27 PAREDES, op. cit., p. 92.

28 Un examen pormenorizado de la relación entre la peste y la conducta moral de los individuos alcanzados por ella en la modernidad temprana puede leerse en ACHINSTEIN, Sharon. Plagues and Publication: Ballads and the Representation of Disease in the English Renaissance. Criticism, vol. 34, n. 1, 1992, pp. 27-49.

29 DEFOE, Diario del año..., op. cit., p. 102. Un poco antes, el narrador señala: “Nada más que la intervención inmediata de la Mano Divina, nada más que su omnipotencia, pudo operar semejante cambio. El contagio desafiaba todo remedio; la muerte hacía estragos por doquier; si las cosas hubieran continuado durante algunas semanas más, la ciudad habría quedado desnuda de todo cuanto poseía alma." "En ese mismo momento, ... quiso Dios, para nuestra grande y dulcísima sorpresa, abatir la furia del mal..." (p. 101). 
Pocos párrafos después, deslegitima el poder curativo de los médicos y los recursos por ellos utilizados cuando agrega:

\begin{abstract}
No era el efecto de una medicina recientemente hallada, ni el descubrimiento de una nueva cura, ni el resultado de una experiencia operatoria obtenida por los médicos. Era, evidentemente, un efecto de la Mano invisible de Aquel que trabaja en secreto y que primeramente había desencadenado la enfermedad sobre nosotros como un juicio. ${ }^{30}$
\end{abstract}

En un mundo en proceso de secularización, desde la perspectiva de un escritor formado en el puritanismo como lo era Defoe, la peste sólo podía ser comprendida en función del juicio y castigo divinos. En ese sentido, aún si el texto también revela las tensiones entre tradición y modernidad o entre comunidad e individuo, tan típicas de este período, para Defoe el fin de la epidemia es explicado a partir del factor religioso. Ahora bien, si en este punto existe una diferencia sustancial respecto de sociedades contemporáneas ya secularizadas -o camino a ello, resulta válido reflexionar sobre las posibles semejanzas entre la experiencia de la plaga londinense y las vivencias actuales producto de la pandemia.

El dilema entre mantener la economía o proteger la vida se plantea desde los inicios del diario apócrifo. Verdadera o falsa, la dicotomía es presentada en las primeras páginas del libro cuando el protagonista se ve enfrentado a tomar una decisión que favorezca sus negocios o proteja su salud:

Tenía ante mí dos importantes asuntos: uno era sostener mi tienda y mis negocios, que eran considerables, y en los cuales había embarcado todo lo que poseía en el mundo; el otro era la protección de mi vida ante una calamidad tan funesta como la que yo vería caer ostensiblemente sobre la ciudad entera. ${ }^{31}$

Ha sido señalado ya que el protagonista es un talabartero y por lo tanto un comerciante relativamente próspero que, frente a la oportunidad de dejar la ciudad y protegerse de la plaga decide en cambio permanecer en Londres. $^{32}$ A lo largo del libro, vuelve sobre el asunto que, lejos de obe-

30 Ibid., p. 102.

31 Ibid., p. 6.

32 Quienes podían, huían de la ciudad por recomendación médica, pues se creía que la peste se transmitía a través del contacto humano y por lo tanto debía evitarse tanto cuanto fuera posible. ACHINSTEIN, op. cit., pp. 33-4. 
decer a una elección personal, determina el comportamiento de todos los ciudadanos de Londres. Así, Defoe describe cómo los habitantes más pobres se vieron forzados a salir a las calles para obtener su sustento $y$, al hacerlo, se arriesgaron a contraer la enfermedad. A fin de cuentas, las consecuencias económicas que la peste podía generar en una ciudad como Londres eran conocidas por todos sus habitantes. ${ }^{33}$ Además de la pérdida de vidas humanas en términos generales (más de 100.000 en este caso), el protagonista constata que, en la medida en que la enfermedad se propagaba, caía ante ella personal dedicado a funciones de primera necesidad que también hacían a la prosperidad y buen funcionamiento de la ciudad. Por una cuestión de proximidad, fueron víctimas de la peste los médicos y sepultureros pero también, en la medida que el contagio avanzó, los carniceros y proveedores de alimentos e insumos básicos.

El aislamiento como forma de prevenir el contagio también es presentado desde el comienzo del diario como el único recurso posible para aquellos que permanecieron en la ciudad o intentaron salir de ella poco después de iniciada la peste. Único antídoto contra las pestes desde la Antigüedad clásica hasta la primera modernidad (e incluso hoy día), ${ }^{34}$ la conciencia sobre la importancia del aislamiento preventivo para mantenerse sano se vuelve evidente cuando el anónimo $\mathrm{H}$. F. señala:

Nuevamente debo observar que la necesidad de salir de las casas para comprar provisiones fue, en gran medida, la ruina de nuestra ciudad, pues en tales ocasiones las personas se contaminaban unas a otras, y hasta las provisiones quedaban a menudo infectadas. ${ }^{35}$

Esto no impide, sin embargo, que su curiosidad lo lleve a caminar por las calles desiertas y hasta las fosas comunes, episodios en los cuales en calidad de "testigo presencial" relata innumerables situaciones de desesperación, muerte y locura.

33 DELUMEAU, op. cit., p. 72: "Porque, para una ciudad, la cuarentena significaba dificultades de avituallamiento, hundimiento de los negocios, paro, desórdenes probables en la calle, etc."

34 Ibid., p. 162.

35 DEFOE, Diario del año..., op. cit., p. 40. 
Por otra parte, Defoe reconoce que la peste no castiga con la misma fuerza a ricos y a pobres. En ese sentido, hace referencia a los sectores de la población más fuertemente alcanzados por la plaga y las precauciones mantenidas en su trato cotidiano con ellos. Así, por ejemplo, explica cuál es el protocolo de seguridad empleado al ir al mercado:

Es verdad que se tomaban todas las precauciones posibles. Cuando alguien compraba un trozo de carne, no tomaba éste de manos del carnicero, sino que directamente lo sacaba del gancho. Y por otra parte el carnicero no tocaba el dinero: lo hacía depositar en un pote lleno de vinagre, destinado a ese uso.

La aplicación de tales medidas, sin embargo, no estaba al alcance del común de la población y Defoe se encarga de destacar las diferencias en las formas de lidiar con la peste según la condición de clase. En más de una ocasión, tras describir las medidas preventivas empleadas por las clases más pudientes, el autor aclara que no todos disponían de los recursos necesarios para hacer frente a la epidemia: "Pero los pobres no disponían de ninguno de tales medios y corrían todos los riesgos." 36

A la vez, un "rasgo de actualidad" del Diario (al menos en relación con cómo algunos países del Cono Sur han lidiado con la actual pandemia en términos discursivos), es la comparación de la peste con una guerra. En el caso de la plaga de 1665 , sin embargo, no se trató de un recurso retórico destinado a aunar esfuerzos y gastos en la lucha contra un enemigo invisible sino del ya mencionado abandono de las costumbres y ritos fúnebres propios de los tiempos de paz. Sin duda, aquella desacralización de la muerte, que en términos de Delumeau la vuelve "anónima y colectiva", fue y continúa siendo uno de los rasgos más sobresalientes del poder disruptivo de la peste en sociedades organizadas. ${ }^{37}$ El "abandono de los ritos tranquilizadores que acompañan en tiempo normal la salida de este mundo" 38 ha sido, en los últimos meses, objeto de reclamo por parte de intelectuales y asociaciones humanitarias además de un tema recurrente en los medios. ${ }^{39}$

36 Ibid., p. 40.

37 DELUMEAU, op. cit., p. 181.

38 Ibid., p. 183.

39 Un claro ejemplo de ello es "Pandemia moderna y piedad antigua: que nos devuelvan 
En lo que atañe a las proximidades entre la experiencia de la peste temprano moderna y nuestras vivencias actuales, merece ser resaltado el hecho de que Defoe percibe claramente la discordancia entre el número de muertes oficiales y las muertes reales. Esto es señalado a comienzos del diario, cuando de forma cuasi periodística el protagonista comienza a sumar las sub-notificaciones al número oficial de óbitos y compararlas con la cantidad de entierros que constata están ocurriendo en las parroquias de la ciudad (Figura 3). De tal forma, el anónimo H. F. señala:

Debo añadir que si el registro obituario denunciaba 5000, en realidad debía de haber dos o tres veces más. No era posible creer en la exactitud de las cifras, porque aquella situación no era la más indicada para llevar un registro estricto en medio de la confusión reinante. ${ }^{40}$

Ahora bien, a diferencia de la sospecha que despiertan las notificaciones de muertes hechas por las autoridades en Preparativos oportunos para una peste ${ }^{41}$ en el caso de Diario la variación entre el número de muertes oficiales y las sub-notificaciones encuentra su explicación en la imposibilidad de las autoridades locales para dar cuenta del número de óbitos a medida que la cantidad de casos aumenta.

Por último, sobre el papel de los medios, resulta de interés señalar que, aún en sus inicios, la prensa permitió la multiplicación de experiencias y el crecimiento de una esfera pública en la que circularon información y vivencias de la peste. Esto no solo expuso un conjunto de experiencias individuales en torno a la peste ante un público masivo sino que habilitó, como en el caso de Defoe, el uso literario de dichas vivencias. En la actualidad, el uso de redes sociales y otros medios de comunicación, acaso una prolongación exacerbada de aquella esfera pública temprano-moderna, también ha

la milenaria humanidad con nuestros enfermos y muertos", nota publicada por José Emilio Burucúa en el suplemento de cultura del periódico argentino Infobae el 25 de mayo de 2020. Disponible en: https://www.infobae.com/cultura/2020/05/25/pandemia-moderna-y-piedad-antigua-que-nos-devuelvan-la-milenaria-humanidad-con-nuestros-enfermos-y-muertos/Acceso: 28 de septiembre de 2020.

40 DEFOE, Diario del año..., op. cit., p. 64.

41 PERALDO, Emmanuelle. Telling Figures and Telling Feelings: The Geography of Emotions in the London of Defoe's Journal of the Plague Year and Due Preparations for the Plague (1722). XVII-XVIII [En línea], n. 69, 2012, parr. 8. Disponible en: https://journals. openedition.org/1718/617 Acceso: 30 de junio de 2020. 
Figura 3

Frontispicio de un acta de muertes para Londres entre 1664 y 1665 , período en el que se desarrolló la gran plaga Fuente: Oxford Science Archive/Print Collector/Getty Images

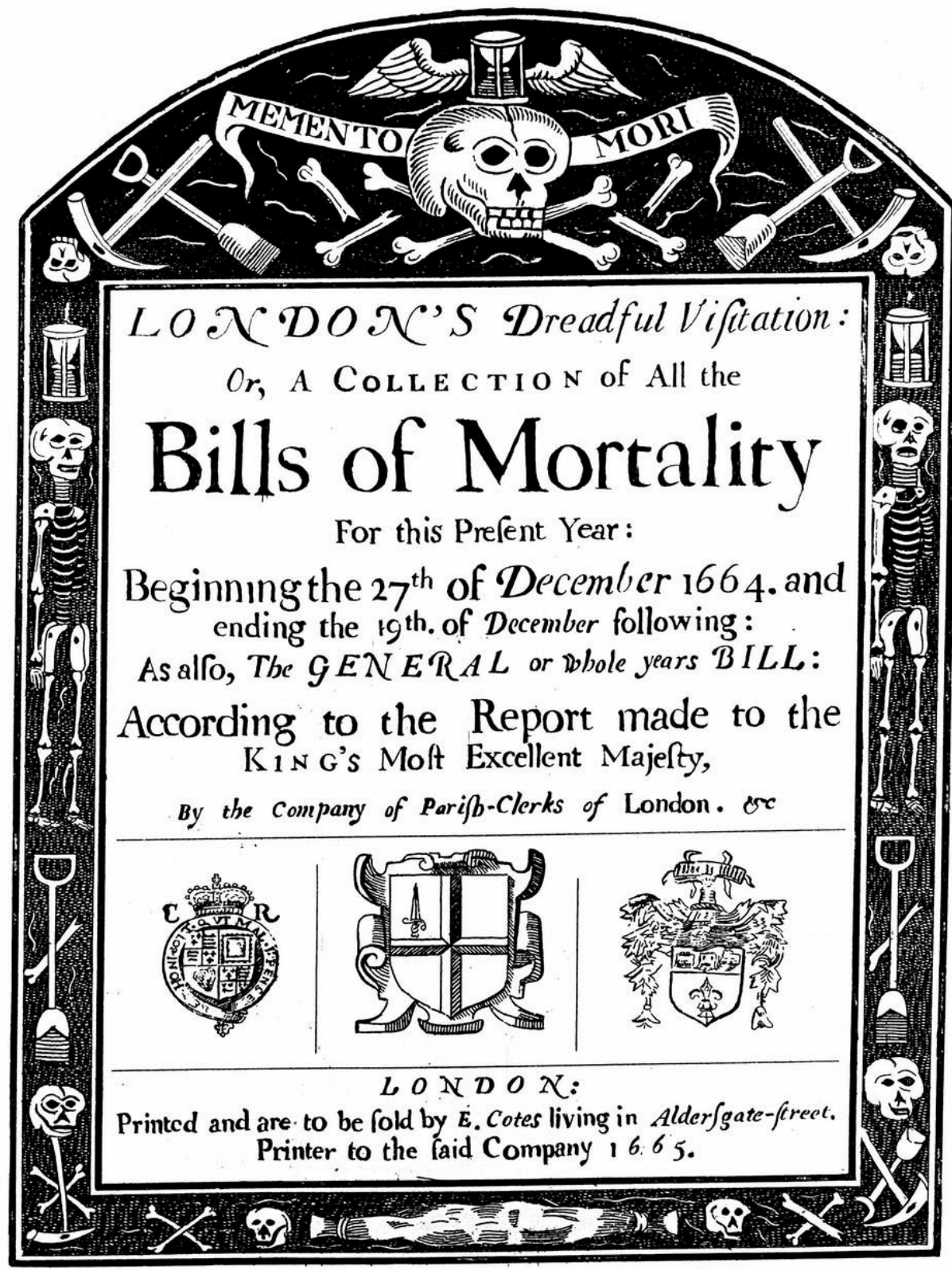

hecho que nuestra experiencia de la pandemia se encuentre atravesada por millares de otras experiencias, que interpretamos como escenarios posibles o futuros probables de nuestro propio presente y con los cuales también generamos empatía.

A modo de conclusión, resta señalar que el extrañamiento provocado por la peste sobre los hábitos y la naturaleza de nuestras costumbres también pareciera ser una condición compartida por todas ellas. Sin duda, la desesperación, la perplejidad y la incapacidad de pensar en un futuro o 
proyectar más allá de nuestro presente que las pestes, plagas o epidemias provocan, son formas extremas de reflexionar sobre la vida y el sentido de nuestras prácticas cotidianas. ${ }^{42}$ Resulta necesario pensar cuánto de esa reflexión profunda provoca como contrapartida la búsqueda desesperada de volver a la "normalidad".

Recebido e aceito em 23 de março de 2021.

Este é um artigo publicado em acesso aberto sob uma licença Creative Commons (cc) $\mathbf{B r}$

42 PAREDES, op. cit., p. 91. DELUMEAU, op. cit., p. 183. 